\title{
A Two-Point Boundary Value Problem by Using a Mixed Finite Element Method
}

\author{
Pedro Pablo Cárdenas Alzate, José Rodrigo González Granada \\ Department of Mathematics, Universidad Tecnológica de Pereira, Pereira, Colombia \\ Email: ppablo@utp.edu.co, jorodryy@utp.edu.co
}

Received 9 October 2015; accepted 15 November 2015; published 18 November 2015

Copyright (C) 2015 by authors and Scientific Research Publishing Inc.

This work is licensed under the Creative Commons Attribution International License (CC BY). http://creativecommons.org/licenses/by/4.0/

(c) (i) Open Access

\section{Abstract}

This paper describes a numerical solution for a two-point boundary value problem. It includes an algorithm for discretization by mixed finite element method. The discrete scheme allows the utilization a finite element method based on piecewise linear approximating functions and we also use the barycentric quadrature rule to compute the stiffness matrix and the $L_{2}$-norm.

\section{Keywords}

Two-Point BVP, Galerkin's Method, Non-Symmetric Problem

\section{Introduction}

Finite element methods in which two spaces are used received the domination of mixed finite element method. Sometimes a second variable is introduced in the formulation of the problem by its physical study, for example in the case of elasticity equations and also the Stokes equations where the mixed formulation is the natural one. The mathematical analyses of mixed finite element have been widely developed in the seventies. A general analysis was first developed by [1]. We also have to mention to [2] and [3] which introduced of the fundamental ideas for the analysis of mixed finite elements. We also refer to [4] and [5] where general results are obtained.

An outline of the paper is as follows. We derive the mixed variational formulation for bilinear form nonsymmentric problem and we define the related discrete elements and the error analysis of the associated finite element method is made [6]. We generalize the results to mixed methods using rectangular elements and we use the barycentric quadrature rule to compute the stiffness matrix, the load vector and the $L_{2}$-norm. Finally, numerical experiments are given to illustrate the present theory [7]. 


\section{Error in the Finite Element Solution}

Recall that $H_{0}^{1}$ in one dimension by Sobolev's inequality, so that $I_{h} v$ is defined for $v \in H_{0}^{1}$. We can prove that with $\|v\|_{K_{j}}=\|v\|_{L_{2}\left(K_{j}\right)}$ and $|v|_{2, K_{j}}=|v|_{H^{2}\left(K_{j}\right)}$

$$
\left\|I_{h} v-v\right\|_{K_{j}} \leq C h_{j}^{2}|v|_{2, K_{j}}
$$

and

$$
\left\|\left(I_{h} v-v\right)^{\prime}\right\|_{K_{j}} \leq C h_{j}|v|_{2, K_{j}}
$$

In fact, by definition we have

$$
I_{h}\left(Q_{1} v\right)=Q_{1} v
$$

where $Q_{1} v$ is the polynomial of degree 1 approximating $v$ in $\left[x_{j-1}, x_{j}\right]$, then we have

$$
\begin{aligned}
& \left\|I_{h} v\right\|_{C\left(K_{j}\right)} \leq\|v\|_{C\left(K_{j}\right)} \\
& \left\|I_{h}-v\right\|_{C\left(K_{j}\right)}=\left\|I_{h}\left(v-Q_{1} v\right)+\left(Q_{1} v-v\right)\right\|_{C\left(K_{j}\right)} \leq 2\left\|v-Q_{1} v\right\|_{C\left(K_{j}\right)} \\
& \left\|v-Q_{1} v\right\|_{C\left(K_{j}\right)} \leq \max _{x \in K_{j}} \int_{K_{j}}\left|x-y \| v^{\prime \prime}(y)\right| \mathrm{d} y
\end{aligned}
$$

therefore, the error is of order $\mathcal{O}\left(h^{2}\right)$ because the fitting is until the second derivative, then

$$
\begin{aligned}
& \left\|I_{h}-v\right\|_{C\left(K_{j}\right)} \leq\left\|v-Q_{1} v\right\|_{C\left(K_{j}\right)} \leq C h_{j}^{2} \int_{K_{j}}\left|v^{\prime \prime}(y)\right| \mathrm{d} y \\
& \left\|I_{h}-v\right\|_{C\left(K_{j}\right)} \leq C h_{j}^{2}|v|_{2, K_{j}}
\end{aligned}
$$

Now, we know that

$$
\left(I_{h} v\right)^{\prime}(x)-v^{\prime}(x)=\frac{1}{h_{j}} \int_{K_{j}}\left(v^{\prime}(y)-v^{\prime}(x)\right) \mathrm{d} y
$$

So, we can write $v^{\prime \prime}$ as

$$
v^{\prime \prime}=\frac{1}{h_{j}}\left(v^{\prime}(y)-v^{\prime}(x)\right)
$$

then we obtain

$$
v^{\prime}(y)-v^{\prime}(x)=h_{j} v^{\prime \prime}
$$

So

$$
\left(I_{h} v\right)^{\prime}(x)-v^{\prime}(x)=\frac{1}{h_{j}} h_{j} \int v^{\prime \prime} \mathrm{d} y=\int v^{\prime \prime} \mathrm{d} y
$$

To write the norm in $L_{2}$, fort take square

$$
\left(\left(I_{h} v\right)^{\prime}(x)-v^{\prime}(x)\right)^{2}=\left(\int v^{\prime \prime} \mathrm{d} y\right)^{2}
$$

Next, integrate with respect to $\mathrm{d} x$ we have:

$$
\int\left(\left(I_{h} v\right)^{\prime}(x)-v^{\prime}(x)\right)^{2} \mathrm{~d} x=\int\left(\int v^{\prime \prime} \mathrm{d} y\right)^{2} \mathrm{~d} x=\int\left(v^{\prime \prime}\right)^{2}\left(\int \mathrm{d} y\right)^{2} \mathrm{~d} x=h_{j}^{2} \int\left(v^{\prime \prime}\right)^{2} \mathrm{~d} x
$$

Taking the square root finally we obtain

$$
\sqrt{\int\left(\left(I_{h} v\right)^{\prime}(x)-v^{\prime}(x)\right)^{2} \mathrm{~d} x}=h_{j} \sqrt{\int\left(v^{\prime \prime}\right)^{2} \mathrm{~d} x} \leq C h_{j}|v|_{K_{j}}
$$




$$
\left\|\left(I_{h} v-v\right)^{\prime}\right\|_{K_{j}} \leq C h_{j}|v|_{2, K_{j}}
$$

\section{Galerkin's Method}

Galerkin's method: Let $a(\cdot, \cdot)$ and $L(\cdot)$ satisfy the assumptions of the Lax-Milgram lemma

$$
\begin{aligned}
& |a(v, w)| \leq C_{1}\|v\|_{V}\|w\|_{V}, \quad \forall v, w \in V, \\
& a(v, v) \geq C_{2}\|v\|_{V}^{2}, \quad \forall v \in V, \\
& |L(v)| \leq C_{3}\|v\|_{V}, \quad \forall v \in V,
\end{aligned}
$$

and $u \in V$ be the solution of $a(u, v)=L(v)$. Let $\hat{V} \in V$ be a finite-dimensional subspace and $\hat{u} \in \hat{V}$ be determined by Galerkin's method: $a(\hat{u}, v)=L(v)$ for all $v \in \hat{V}$. We want to prove that

$$
\|\hat{u}-u\|_{V} \leq \frac{C_{1}}{C_{2}} \min _{\mathcal{X} \in \hat{V}}\|\mathcal{X}-u\|_{V}
$$

and with $a(\cdot, \cdot)$ symmetric,

$$
\begin{aligned}
& \|\hat{u}-u\|_{a}=\min _{\mathcal{X} \in \hat{V}}\|\mathcal{X}-u\|_{a} \\
& \|\hat{u}-u\|_{V} \leq \sqrt{\frac{C_{1}}{C_{2}}} \min _{\mathcal{X} \in \hat{V}}\|\mathcal{X}-u\|_{V}
\end{aligned}
$$

In fact, let $a(\hat{u}, \mathcal{X})=L(\mathcal{X})$ and $a(u, \mathcal{X})=L(\mathcal{X})$ with $\mathcal{X} \in \hat{V}, \hat{u} \in \hat{V}, u \in V$ and $\hat{V} \subset V$. Thus,

$$
a(\hat{u}-u, \mathcal{X})=0 \quad \forall \mathcal{X} \in \hat{V}
$$

Now, from the assumptions of the Lax-Milgram lemma we have

$$
\|\hat{u}-u\|_{V}^{2} \leq \frac{1}{C_{2}} a(\hat{u}-u, \hat{u}-u)=\frac{1}{C_{2}} a(\hat{u}-u, \mathcal{X}-u),
$$

$(u-\mathcal{X}) \in \hat{V}$. And

$$
a(\hat{u}-u, \mathcal{X}-u) \leq C_{1}\|\hat{u}-u\|_{V}\|\mathcal{X}-u\|_{V}
$$

Divide by $C_{2}$ both sides we have

$$
\frac{1}{C_{2}} a(\hat{u}-u, \mathcal{X}-u) \leq \frac{C_{1}}{C_{2}}\|\hat{u}-u\|_{V}\|\mathcal{X}-u\|_{V}
$$

Now, using (5), we get that

$$
\|\hat{u}-u\|_{V}^{2} \leq \frac{1}{C_{2}} a(\hat{u}-u, \mathcal{X}-u) \leq \frac{C_{1}}{C_{2}}\|\hat{u}-u\|_{V}\|\mathcal{X}-u\|_{V}
$$

Finally, we can prove (4)

$$
\|\hat{u}-u\|_{V} \leq \frac{C_{1}}{C_{2}} \min _{\mathcal{X} \in \hat{V}}\|\mathcal{X}-u\|_{V}
$$

Now, for the symmetric $a(\cdot, \cdot)$ we can apply Riesz representation theorem. Therefore the norm of the inner product can be written as

$$
a(v, v)^{1 / 2}=\|v\|_{a}
$$

Similar to previous proof, we have

$$
a(\hat{u}-u, \mathcal{X})=0 \quad \forall \mathcal{X} \in \hat{V}
$$




$$
\|\hat{u}-u\|_{a}^{2}=a(\hat{u}-u, \hat{u}-u)=a(\hat{u}-u, \mathcal{X}-u)
$$

Therefore

$$
\|\hat{u}-u\|_{a}=\min _{\mathcal{X} \in \hat{V}}\|\mathcal{X}-u\|_{a}
$$

As for the norm in $V$, we have

$$
\begin{aligned}
& \|\hat{u}-u\|_{V}^{2} \leq \frac{1}{C_{2}} a(\hat{u}-u, \hat{u}-u)=\frac{1}{C_{2}} a(\hat{u}-u, \mathcal{X}-u), \\
& \|\hat{u}-u\|_{V} \leq \frac{1}{\sqrt{C_{2}}}(a(\hat{u}-u, \mathcal{X}-u))^{1 / 2}
\end{aligned}
$$

From the assumptions we obtain

$$
|a(v, w)| \leq C_{1}\|v\|_{V}\|w\|_{V} \quad \forall v, w \in V
$$

With $v=\hat{u}-u$ and $w=\mathcal{X}-u$ we have

$$
(|a(\hat{u}-u, \mathcal{X}-u)|)^{1 / 2} \leq \sqrt{C_{1}}\left(\|\hat{u}-u\|_{V}\|\mathcal{X}-u\|_{V}\right)^{1 / 2}
$$

Using this inequality, (6) becomes

$$
\|\hat{u}-u\|_{V} \leq \frac{1}{\sqrt{C_{2}}}(a(\hat{u}-u, \mathcal{X}-u))^{1 / 2} \leq \sqrt{\frac{C_{1}}{C_{2}}}\left(\|\hat{u}-u\|_{V}\|\mathcal{X}-u\|_{V}\right)^{1 / 2}
$$

We know that

$$
\|\hat{u}-u\|_{V} \leq\|\mathcal{X}-u\|_{V}
$$

from Equation (4) which was proven in the previous section, therefore

$$
\begin{aligned}
& \|\hat{u}-u\|_{V} \leq \sqrt{\frac{C_{1}}{C_{2}}}\left(\|\hat{u}-u\|_{V}\|\mathcal{X}-u\|_{V}\right)^{1 / 2} \leq \sqrt{\frac{C_{1}}{C_{2}}}\left(\|\mathcal{X}-u\|_{V}^{2}\right)^{1 / 2} \\
& \|\hat{u}-u\| \leq \sqrt{\frac{C_{1}}{C_{2}}} \min _{\mathcal{X} \in \hat{V}}\|\mathcal{X}-u\|_{V}
\end{aligned}
$$

\section{FEM for Bilinear Form Non-Symmetric Problem}

We consider the problem

$$
-\nabla \cdot(a \nabla u)+b \cdot \nabla u+c u=f
$$

in $\Omega$ with $u=0$ on $\Gamma$. A finite element method for this problem with an error bound in the $H^{1}$-norm is as follows. First we need to find the variational formulation for this problem. In fact, multiply by a function $\phi \in C_{0}^{1}$ on $\Omega$ :

$$
-\nabla \cdot(a \nabla u) \phi+b \cdot \nabla u \phi+c u \phi=f \phi
$$

Next, integrate over the domain $\Omega$ :

$$
\int_{\Omega}(-\nabla \cdot(a \nabla u) \phi+b \cdot \nabla u \phi+c u \phi) \mathrm{d} x=\int_{\Omega}(f \phi) \mathrm{d} x
$$

Now, the left hand side can be written using integrating by parts:

$$
\int_{\Omega}((a \nabla u) \nabla \phi+b \cdot \nabla u \phi+c u \phi) \mathrm{d} x=\int_{\Omega}(f \phi) \mathrm{d} x
$$

Therefore we have the bilinear form

$$
a(\phi, u)=\int_{\Omega}((a \nabla u) \nabla \phi+b \cdot \nabla u \phi+c u \phi) \mathrm{d} x
$$


and the linear functional

$$
L(\phi)=(\phi, f)=\int_{\Omega}(f \phi) \mathrm{d} x
$$

The space $C_{0}^{1}$ is dense in $H^{1}$ and by Lax Milgram theorem, there is a weak solution in $H^{1} . a(\cdot, \cdot)$ is coercive in $H^{1}$, therefore

$$
a(v, v) \geq \min _{x \in \Omega} a(x)\left\|v^{\prime}\right\|^{2} \geq \frac{a}{2}\|v\|_{1}^{2} \quad \forall v \in H^{1}
$$

The bilinear form is also bounded:

$$
|a(v, w)| \leq C\|v\|_{1}\|w\|_{1} \quad \forall v, w \in H^{1}
$$

Now, we would like to minimize the residual

$$
\|A u-f\|=\min _{\phi \in V_{h}}\|A \phi-f\|^{2}
$$

Also we have (see [2])

$$
|L(V)| \leq\|f\|\|v\| \quad \forall v \in H^{1}
$$

where $C_{3}=\|f\|$. Therefore, we can apply the previously proven statement (2) in order to estimate a bound for the error

$$
\|\hat{u}-u\|_{H}^{1} \leq \frac{C_{1}}{C_{2}} \min _{\mathcal{X} \in H^{1}}\|\mathcal{X}-u\|_{H^{1}}
$$

which for our case become

$$
\|\hat{u}-u\|_{H}^{1} \leq \frac{2 C}{a} \min _{\mathcal{X} \in H^{1}}\|\mathcal{X}-u\|_{H^{1}}
$$

\section{BVP by Finite Element Method}

We consider the boundary value problem



Figure 1. Mesh used to solve the problem (7) by using (8). 


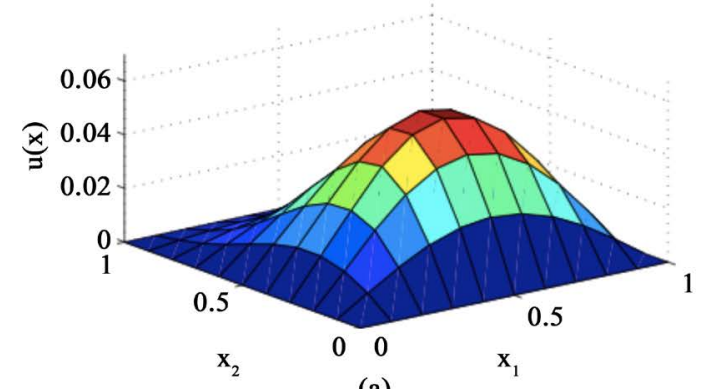

(a)

Boundary Value Problem (5.18), $h=1 / 20$

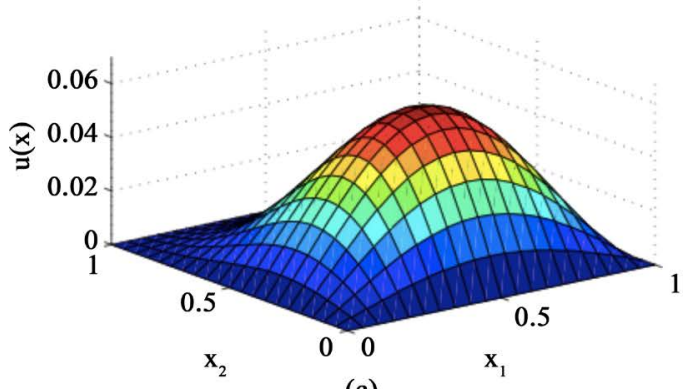

(c)



(b)

Error: (Exact-Numerical) Solution, $h=1 / 20$

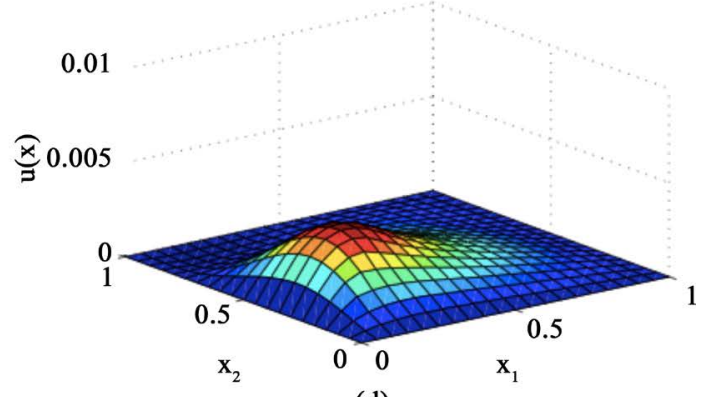

(d)

Figure 2. (a) Solution of the system (7) using $h=\frac{1}{10}$; (b) Error of the approximation in (a), compared to the real solution; (c) Solution of the system (7) using $h=\frac{1}{20}$; (d) Error of the approximation in (b), compared to the real solution.

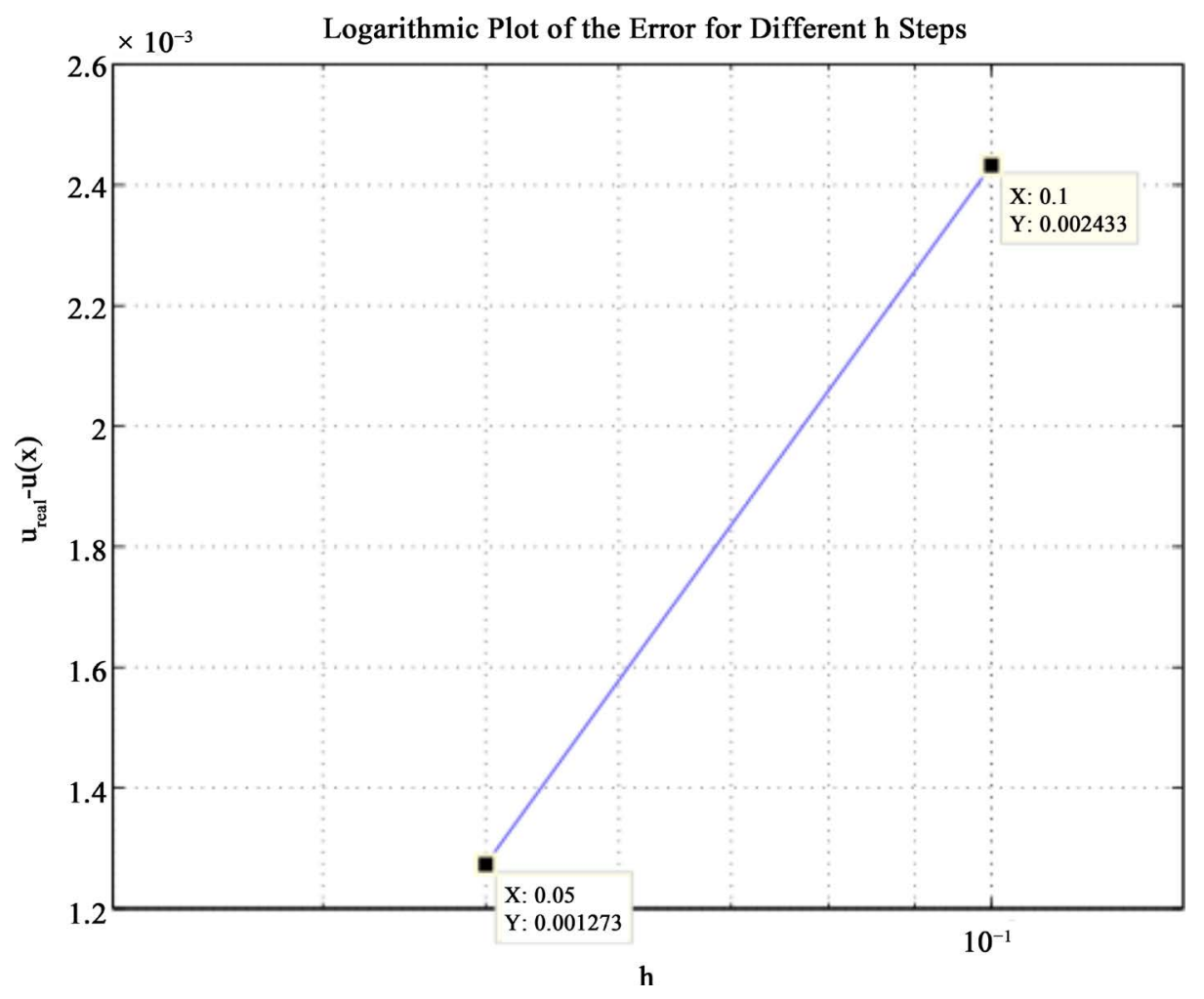

Figure 3. Logarithmic plot of the $L_{2}$-norm of the error vs. the choice of $h$. 


$$
-\Delta u=f(x)=\sin \left(\pi x_{1}\right) \sin \left(\pi x_{2}\right)+\sin \left(\pi x_{1}\right) \sin \left(2 \pi x_{2}\right)
$$

We want to solve it by the finite element method

$$
a\left(u_{h}, \mathcal{X}\right)=(f, \mathcal{X}) \quad \forall \mathcal{X} \in S_{h}
$$

based on piecewise linear approximating functions on the partition $\Omega=(0,1) \times(0,1)$, divided into triangles by inserting a diagonal with positive slope into each mesh-square with $h=1 / 10,1 / 20$. We will use the barycentric quadrature rule to compute the stiffness matrix, the load vector and the $L_{2}$-norm. The Figure 1 shows the mesh used to solve this problem (system (7)). With this mesh, the stiffness matrix A was computed considering each node, from a total of $(M-1) \times(M-1)$ interior nodes $(M=1 / h$, which $h$ is the step size). The basis function is a set of pyramidal functions. At each node, there are two triangles $\left(\phi_{U 1}, \phi_{U 2}\right)$ coming at a straight angle, and four others coming with an acute angle $\left(\phi_{L 1}, \phi_{L 2}, \phi_{L 3}, \phi_{L 4}\right)$. The basis functions are therefore

$$
\phi_{i}= \begin{cases}\frac{x_{1}}{h}-\frac{x_{2}}{h}+1, & \in U 1, \\ -\frac{x_{1}}{h}+\frac{x_{2}}{h}+1, & \in U 2, \\ \frac{x_{1}}{h}+1, & \in L 1, \\ \frac{x_{2}}{h}+1, & \in L 2, \\ -\frac{x_{2}}{h}+1, & \in L 3, \\ -\frac{x_{1}}{h}+1, & \in L 4\end{cases}
$$

Therefore we obtain

- $\left(\nabla \phi_{i}, \nabla \phi_{i+1}\right)$. There are two triangles common to these neighboring nodes, therefore this inner product is -1 . It is the same for the neighbours on the left $\left(\nabla \phi_{i}, \nabla \phi_{i-1}\right)$ (2 common triangles). Similarly for the neighbours on the rows above and below. This inner product was used in the stiffness matrix A.

- The Barycentric Quadrature Rule were used to evaluate the integral on the right hand side,

$$
f\left(f, \phi_{i}\right)=\sum_{K \in T_{h}} \int_{K} f \phi_{i} \mathrm{~d} x \approx \sum_{K \in T_{h}} q_{K}(f \phi)
$$

where

$$
q_{K}(\phi)=\operatorname{area}(K) \phi\left(\frac{1}{3} \sum_{l=1}^{3} P_{l, K}\right)
$$

where $K$ is each triangle in the mesh, $P_{l, K}$ the vertices.

- Figure 2 shows the solution of (7) for $h=1 / 10$ (panel A) and $h=1 / 20$ (panel C). Compared to the correct solution

$$
u(x)=\frac{\sin \left(\pi x_{1}\right) \sin \left(\pi x_{2}\right)}{2 \pi^{2}}+\frac{\sin \left(\pi X_{1}\right) \sin \left(2 \pi x_{2}\right)}{5 \pi^{2}}
$$

the errors are shown in (panel B) and (panel C) respectively.

- $h \times L_{2}$ norm of this error is shown Figure 3 .

\section{Acknowledgments}

We thank the editor and the referee for their comments and group GEDNOL of the Universidad Tecnológica de Pereira-Colombia. 


\section{References}

[1] Brezzi, F. (1974) On the Existence, Uniqueness and Approximation of Saddle Points Problems Arising from Lagrangian Multipliers. ESAIM: Mathematical Modelling and Numerical Analysis_Modélisation Mathématique et Analyse Numérique, 8, 129-151.

[2] Larsson, S. and Thome, V. (2009) Partial Differential Equations with Numerical Methods. Springer-Verlag, New York.

[3] Canuto, C. and Hussaini, M. (1988) Spectral Methods in Fluids Dynamics. Springer Series in Computational Physics, Springer-Verlag, Berlin. http://dx.doi.org/10.1007/978-3-642-84108-8

[4] Fortin, M. (1977) An Analysis of the Convergence of Mixed Finite Element Methods. R.A.I.R.O, 11, 341-354.

[5] Guzman, J. (2010) A Unified Analysis of Several Mixed Methods for Elasticity with Weak Stress Symmetric. Journal of Scientific Computing, 4, 156-169. http://dx.doi.org/10.1007/s10915-010-9373-2

[6] Yao, C. and Jia, S. (2014) Asymptotic Expansion Analysis of Nonconforming Mixed Finite Element Methods for Time-Dependent Maxwell's Equations in Debye Medium. Applied Mathematics and Computation, 229, 34-40. http://dx.doi.org/10.1016/j.amc.2013.12.016

[7] Pal, M. and Lamine, S. (2015) Validation of the Multiscale Mixed Finite-Element Method. International Journal for Numerical Methods in Fluids, 77, 206-223. http://dx.doi.org/10.1002/fld.3978 